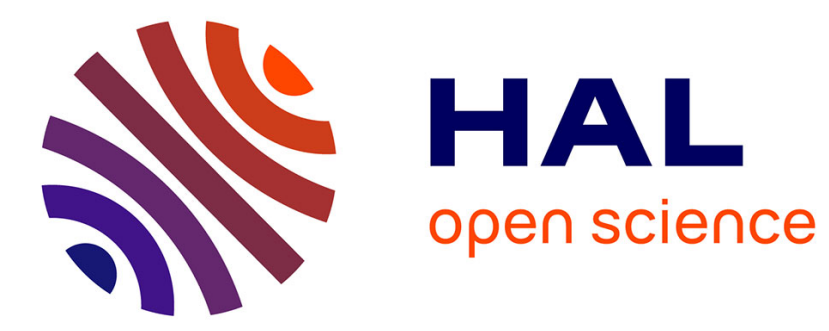

\title{
Single shot measurement of the optical Kerr effect kinetics
}

Gilles Le Saux, François Salin, Patrick Georges, Gérard Roger, Alain Brun

\section{To cite this version:}

Gilles Le Saux, François Salin, Patrick Georges, Gérard Roger, Alain Brun. Single shot measurement of the optical Kerr effect kinetics. Applied optics, 1988, 27 (4), pp.777-779. hal-00691677

\section{HAL Id: hal-00691677 \\ https://hal-iogs.archives-ouvertes.fr/hal-00691677}

Submitted on 26 Apr 2012

HAL is a multi-disciplinary open access archive for the deposit and dissemination of scientific research documents, whether they are published or not. The documents may come from teaching and research institutions in France or abroad, or from public or private research centers.
L'archive ouverte pluridisciplinaire HAL, est destinée au dépôt et à la diffusion de documents scientifiques de niveau recherche, publiés ou non, émanant des établissements d'enseignement et de recherche français ou étrangers, des laboratoires publics ou privés. 


\title{
Single shot measurement of the optical Kerr effect kinetics
}

\author{
G. Le Saux, F. Salin, P. Georges, G. Roger, and A. Brun
}

\begin{abstract}
We present a method allowing measurement of the kinetics of the optical Kerr effect in transparent media using a single femtosecond pulse. This method is based on a spectrotemporal transformation. We describe the experimental results obtained for ultrafast and noninstantaneous responses.
\end{abstract}

The use of femtosecond optical pulses with very high peak power ${ }^{1}$ has resulted in temporal responses of nonlinear processes being attained in various classes of material. ${ }^{2}$ These responses are generally measured with a pump-and-probe technique; unfortunately, this method implies averaging over a great number of successive shots to obtain the whole response. The result then greatly depends on the reproducibility of the laser pulses. Therefore it could be interesting to develop a method giving the complete kinetics using single shot excitation. In this paper we present such a method of measuring with a single pulse the temporal kinetics of the optical Kerr effect in a transparent medium. Uses in ultrafast and noninstantaneous response time measurements are described.

The optical Kerr shutter technique is now widely used with femtosecond pulses because it provides a convenient way to measure, for example, the dynamics of molecular orientation in liquids, electronic state distortions in glasses or other condensed media. In these experiments, an ultrashort pump pulse induces a transient birefringence in the Kerr medium. This medium is placed between crossed polarizer and analyzer, and the shutter transmission is proportional to the index variation squared. The time evolution of the Kerr shutter transmission is then directly related to to the relaxation time of the medium studied. When the relaxation time is very fast compared to the excitation duration, the induced transient birefringence exactly follows the pump pulse shape. ${ }^{3}$ When the pump pulse duration is much shorter than the

The authors are with Universite Paris-Sud, Institut d'Optique Theorique et Appliquée, U.A. 14 CNRS, B.P. 43, 91406 Orsay CEDEX, France.

Received 1 July 1987.

0003-6935/88/040777-03\$02.00/0.

(C) 1988 Optical Society of America relaxation process, the index variation is directly governed by the dynamics of the optical Kerr effect in the medium. Let us now consider the transmission of a very long duration probe pulse through the Kerr shutter. The probe signal temporal shape is modulated by the temporal variations of the Kerr shutter transmission. For an instantaneous response the transmitted probe pulse shape is proportional to the square of the pump shape; for a noninstantaneous response the transmitted probe temporal shape is proportional to the square of the dynamics of the Kerr effect. The problem is to record the temporal shape of the transmitted probe.

Our method is based on an idea introduced by Valdmanis ${ }^{4}$ in his real-time picosecond oscilloscope. The basic principle of these experiments is to use a long (picosecond) chirped probe pulse to transform a temporal modulation into a spectral modulation. A spectrograph then converts the wavelength-encoded temporal information to the spatial domain for readout. Using this spectrotemporal transformation, we obtain the whole Kerr shutter kinetics on a single shot basis.

As a chirped probe pulse we have used a spectral continuum created by focusing an intense femtosecond optical pulse on a water cell. As has already been shown, ${ }^{5}$ this spectral continuum is chirped, that is to say, the different frequencies are distributed along the continum temporal profile. Knowledge of the chirp behavior allows us to easily convert frequency information in the temporal domain.

We have used this method for experimental measurements of optical Kerr effect kinetics in a thoriumdoped glass (ultrafast response time) and in toluene (noninstantaneous response). Figure 1 depicts the experimental arrangement. The intense femtosecond pulse (620-nm wavelength) is split into two beams: the first (pump beam) is directly focused in a Kerr medium placed between crossed polarizer and analyzer; the second (probe beam) is focused first on a water cell to create the spectral continuum. Then the continuum pulse is focused on the Kerr medium with 


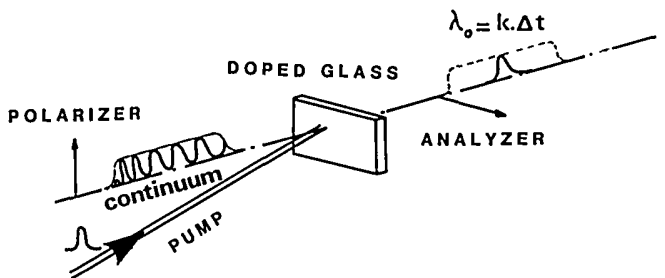

Fig. 1. Experimental setup. The pump and probe beams are focused on the sample placed between crossed polarizer and analyzer. The transmitted probe is dispersed by a spectrograph and detected by an optical multichannel analyzer.

linear polarization. We record the transmitted probe pulse spectrum with a 0.1-m monochromator coupled to an optical multichannel analyzer (OMA). The temporal coincidence between the two pulses can be adjusted with 15 -fs precision.

First, we characterized the continuum pulse chirp. We used, as an ultrafast Kerr shutter, a thorium-doped glass with an instantaneous response. We measured, for each time delay between the pump and the probe pulses, the central wavelength of the transmitted probe pulses. Figure 2 shows the continuum pulse chirp. This result indicates a positive chirp which is much larger than the Fork et al..$^{5}$ experimental results. This difference could come from the fact that Fork et $a l$. used a thin ethylene glycol jet and we used a thick (1-cm) water cell. As shown in Fig. 2, the chirp is nearly linear between 500 and $600 \mathrm{~nm}$ with a slope of $\sim 13 \mathrm{fs} / \mathrm{nm}$. In our optical setup the continuum passes through the water cell, a thick $(1.5-\mathrm{cm})$ Glan polarizer and two lenses ( $\sim 1 \mathrm{~cm}$ thick). If we calculate the chirp introduced by all these dispersive elements, we find (using a dispersion value equal to $370 \mathrm{ps} / \mathrm{nm} \cdot \mathrm{km}$ at a wavelength of $550 \mathrm{~nm}$ ) a chirp coefficient $K$ equal to 13 fs/nm.

This value shows that the continuum chirp is mainly created by the group velocity dispersion introduced by our optical arrangement. This implies that the chirp coefficient can be easily adjusted (by controlling the thickness of the dispersive elements), and that in addition its value only depends a little on the input pulse characteristics and does not need to be determined for each laser shot.

First, we measured the Kerr effect time behavior in a thorium-doped glass. In this experiment we used the spectral continuum probe in a region centered at 570 $\mathrm{nm}$ where the chirp is linear. The probe spectrum in front of the sample is given in Fig. 3. The pump pulse is temporally coincident with that part of the probe centered at $570 \mathrm{n}$. We recorded the spectrum of the probe transmitted by the Kerr shutter (Fig. 4). The time calibration of the $X$ axis is done using the chirp measurements and gives a Kerr shutter response time of $\sim 150 \mathrm{fs}$. We have plotted in the same diagram (Fig. 5) three superimposed pulses each delayed from the previous one by adjusting the fixed delay time between the pump and the continuum probe. This figure shows that each delay corresponds to a different peak wavelength selected in the continuum. In addition,

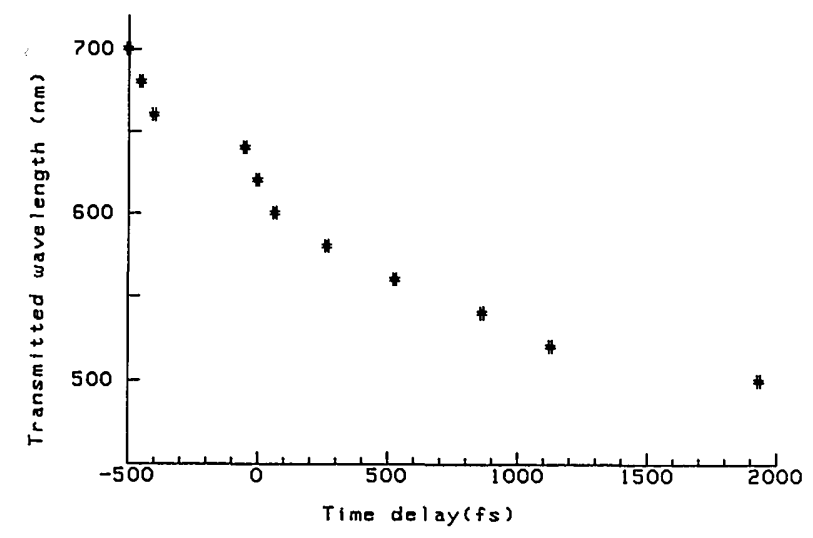

Fig. 2. Continuum chirp measured with the ultrafast Kerr shutter. The chirp is found to be linear between 500 and $600 \mathrm{~nm}$ and equal to $13 \mathrm{fs} / \mathrm{nm}$.

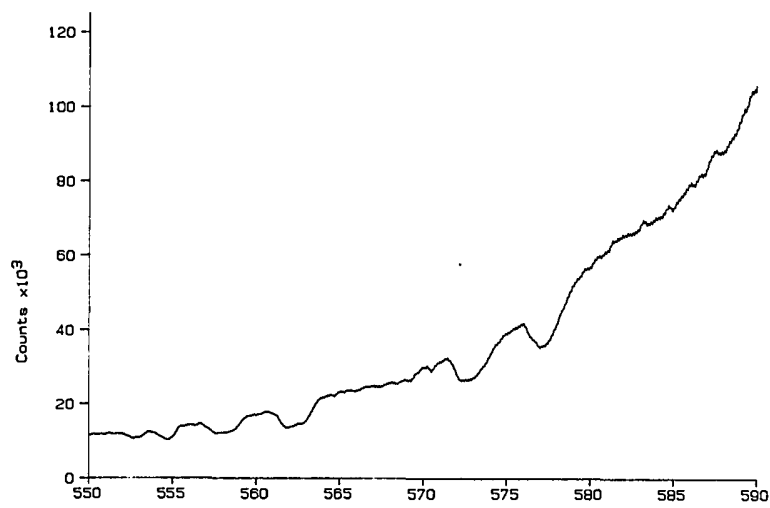

Fig. 3. Probe continuum intensity spectrum in the region of the linear chirp, used for the doped glass characterization.

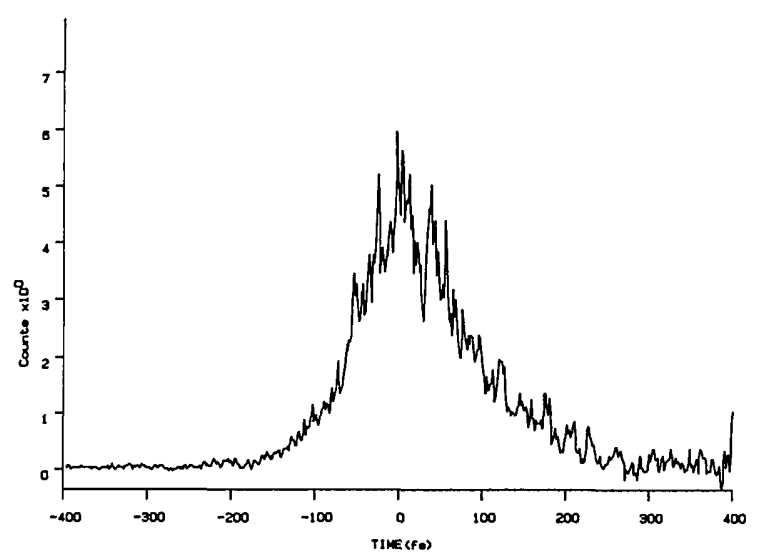

Fig. 4. Real single shot measurement corresponding to an optical Kerr shutter response of 150 , fs.

the measured response depends only a little on the selected central wavelength as long as the chirp remains linear in this domain. In this case (instantaneous response), the kinetics measurement does not give any information about the relaxation time of the medium, but we can obtain, using this single shot method, a good approximation of the femtosecond pulse temporal shape. For example, this method can be used to study and detect dissymmetrical pulse shapes, which is not possible using classical detection autocorrelation methods.

Next, we measured the kinetics of the Kerr effect in 


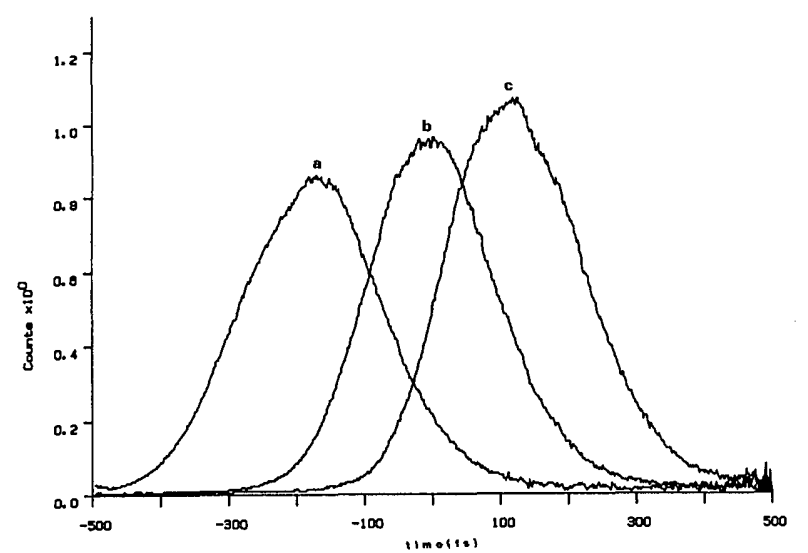

Fig. 5. Different signals obtained for different delays between the pump and the probe (signal averaged over 100 successive shots): $a$, delay $70 \mathrm{fs}$; wavelength $585 \mathrm{~nm} ; b$, delay $250 \mathrm{fs}$; wavelength $570 \mathrm{~nm} ; c$, delay $400 \mathrm{fs}$; wavelength $558 \mathrm{~nm}$.

toluene, using à conventional pump-and-probe technique. We found the existence of two separate relaxation times: the first ultrafast (compared to our pump pulse duration), the other slower and equal to $1.8 \mathrm{ps}$ giving a slow Kerr shutter relaxation time equal to 900 fs. Making the same measurements using the single shot method we also observed two different decay zones. The first part, too fast to be resolved with our pump pulse duration, gives a profile similar to that observed for the doped glass. On the other hand, the slower relaxation due to the molecular reorientation is easily detectable. This slow exponential decay is presented in Fig. 6. The fast part of the response, about ten times greater, is not visible in this figure. The probe spectrum used in this measurement is broader than that used for the doped glass to obtain a window of $\sim 2 \mathrm{ps}$ (varying from 450 to $600 \mathrm{~nm}$ ). We also used a less dispersive monochromator to get the whole spectrum on our detector. The decay constants measured by the two different methods are in good agreement; the comparison between the two detected signals is presented Fig. 7.

We have presented in this paper a method allowing

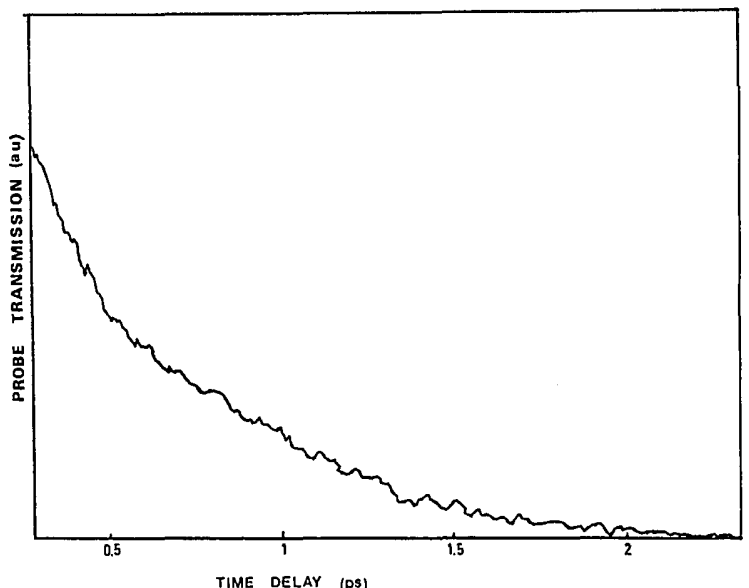

Fig. 6. Typical signal obtained for a noninstantaneous response using the single shot technique: the molecular reorientation of touluene; Evolution for delay times $>350 \mathrm{fs}$ after the probe transmission peak. measurement of the optical Kerr effect kinetics with a subpicosecond resolution using a single shot laser pulse. The principle of this method is to encode temporal information in the wavelength domain using a chirped spectral continuum as the probe. For an instantaneous response time we directly obtain information about the pulse shape. For an exponential decay time, even in the picosecond range we can measure the relaxation time. The main drawback of this method is created by the continuum spectrum amplitude fluctuations. But this problem can be easily solved using two OMA detectors and simultaneously measuring the spectral intensities of both the response and the incident continum. The chirp is mainly created by group velocity dispersion in the water cell used to generate the spectral continuum and in all the glass used in the optical setup. Therefore, the chirp coefficient does not depend on the pump pulse characteristics, and remained constant during our experiments. In particular, it is not necessary to determine the chirp value for each laser shot. In addition the method used to create the chirped probe allows one to obtain adjustable chirp coefficients in a large wavelength domain (varying from 400 to $600 \mathrm{~nm}$ ). In conclusion, this method is easy to use and gives a real-time visualization of the sample temporal relaxation with a subpicosecond resolution.

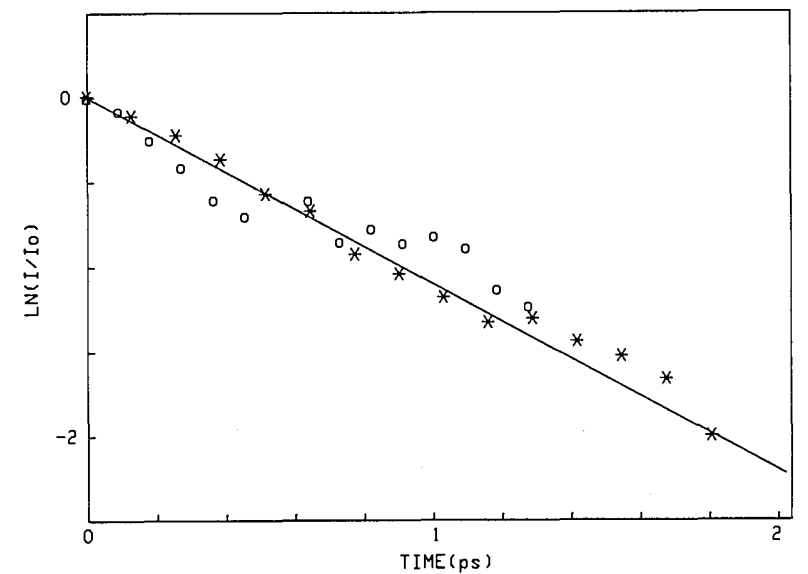

Fig. 7. Comparison of the single shot method with the pump-andprobe technique for noninstantaneous response time. *, single shot method; $O$, conventional pump-and-probe technique. The zero time origin is arbitrarily chosen as $400 \mathrm{fs}$ after the probe transmission peak to clearly discriminate between the two separate components in the response. Kerr shutter relaxation time is $900 \mathrm{fs}$.

\section{References}

1. R. L. Fork, C. V. Shank, and R. Yen, "Amplification of $70 \mathrm{fs}$ Optical Pulse to GW Powers," Appl. Phys. Lett. 41, 223 (1982).

2. A. Migus, A. Antonetti, J. Etchepare, D. Hulin, and A. G. Orszag, "Femtosecond Spectroscopy with High-Power Tunable Optical Pulses," J. Opt. Soc. Am. B2, 584 (1985).

3. K. Sala and M. C. Richardson, "Optical Kerr Effect Induced by Ultrashort Laser Pulses," Phys. Rev. A 12, 1036 (1975).

4. J. A. Valdmanis, "Real Time Picosecond Optical Oscilloscope," in Ultrafast Pehnomena V, G. R. Fleming and A. E. Siegman, Eds. (Springer-Verlag, Berlin, 1986), pp. 82, 83 (1986).

5. R. L. Fork, C. V. Shank, C. Hirlimann, R. Yen, and W. J. Tomlinson, "Femtosecond White-Light Continuum Pulses," Opt. Lett. 8, 1 (1983). 